\title{
Dark Matter: The Source of Space and Time
}

\author{
Keith G. Lyon \\ 16914 Stormy Dr., Haymarket, VA, USA \\ Email: klhaymarket@aol.com
}

Received 17 March 2016; accepted 23 May 2016; published 26 May 2016

Copyright (C) 2016 by author and Scientific Research Publishing Inc.

This work is licensed under the Creative Commons Attribution International License (CC BY).

http://creativecommons.org/licenses/by/4.0/

(c) (i) Open Access

\begin{abstract}
A simple assumption for dark matter leads to magnetic-monopole-like terms to Maxwell's Equations, a photon model with wave-particle duality, nuclear stability, a decelerating expansion of the universe, and a dark-matter relativity that defines the origin of space and time.
\end{abstract}

\section{Keywords}

Dark Matter, Photon, Speed of Light, Nuclear Stability, Space, Time, Superconductivity, Nuclear Fusion, Magnetic Monopole

\section{Introduction}

A century ago, the physics community discarded the theory of an ether being responsible for light propagation. This paper reopens the debate with a specific model for the ether where the ether is the dark matter. The theory specifically assumes that a particle of dark matter has a very small mass, a magnetic charge, and is distributed throughout the universe.

Dark matter is needed to explain the motions of galaxies and is believed to constitute a large fraction of the mass of the universe. A dark-matter particle has never been detected and the only fact known is that it has mass. Several theories have been suggested to define dark matter and this paper adds one more to the list.

The basis of electricity and magnetism is that a charge produces an electric field and a moving charge produces a magnetic field. The theory presented here assumes an additional step, the addition of a magnetic charge, specifically that an electric charge produces an electric field, a moving electric charge moves a magnetic charge, and a moving magnetic charge produces a magnetic field. An immediate observation is that in a sea of magnetic charges, a wave would produce an oscillating magnetic field. From Faraday's Law, an oscillating magnetic field produces an orthogonal oscillating electric field. These two fields resemble the fundamental nature of a photon. With the additional assumption that dark matter is spread throughout the universe, several physical phenomena have more intuitive explanations. 


\section{Theory}

The theory is very simple and assumes that magnetic fields are proportional to the magnetic charge velocity. Thus magnetic charge is undetectable at rest and its motion is detected as a magnetic field, which also explains why dark matter has never been detected in particle collisions since any dark matter generated would blend into the magnetic fields present in particle accelerators. Specifically, the magnetic field $\boldsymbol{B}(\boldsymbol{r})$ is given by:

$$
\boldsymbol{B}(\boldsymbol{r})=b \frac{\boldsymbol{v}(\boldsymbol{r})}{c}
$$

where $\boldsymbol{v}(\boldsymbol{r})$ is the velocity of the magnetic charge at location $\boldsymbol{r}, c$ is the speed of light at that location, and $b$ is magnetic charge (in units of magnetic field). (The addition of $c$ is introduced to avoid creating another unit for magnetic charge.) A key observation about $b$ is that its value must be small for dark matter, otherwise magnetic fields would be observed by relative motion through dark matter.

All of the known physics associated with electricity and magnetism remains unchanged. However, the creation and annihilation of magnetic charge (dark matter) requires additional terms to be added to Maxwell's Equations. As magnetic charge (dark matter) flows into a location where it will be annihilated, magnetic fields that flow into that location are generated and would resemble a "magnetic monopole." Likewise, when magnetic charge (dark matter) is created, magnetic field lines would flow out of that location as this magnetic charge exits. Hence, Maxwell's Equations would need to be modified for the so-called "magnetic monopole" but the "magnetic monopole" is not a particle but a location where annihilation and creation of magnetic charge (dark matter) occurs.

\section{Photon}

The most obvious ramification of this theory is that a simple vibration being transmitted through dark matter would produce an oscillating magnetic field, and from Faraday's Law an oscillating orthogonal electric field. These fields have the same fundamental characteristics of a photon.

Developing a model for the photon is possible, in fact many models can be hypothesized. Any model can be used to calculate the energy in the magnetic and electric fields and compare this energy to the known energy of a photon, $h f$. In addition, any plausible model would need to exhibit the well-known particle-wave duality of the photon.

Several models were considered starting with a simple string of vibrating dark matter. In the case of the single string, either $b$ was too large or the maximum velocity of dark matter exceeded the speed of light by several orders of magnitude. To obtain a model that had reasonable values for magnetic charge and dark-matter velocities well below the speed of light, the photon needed to be rather large compared to the wavelength. This is actually good in that it is consistent with diffraction from a dual slit when the slits are widely separated.

The model assumed here is based on a single string of dark matter at its center that as it vibrates it affects its nearest neighbors which in turn affects its nearest neighbors, etc. This center string has the largest vibration amplitude and the amplitudes of its neighbors are smaller the further away they are. The displacement and velocity for a simple harmonic oscillator are given by $A \sin (\omega t)$ and $A \omega \cos (\omega t)$, where $\omega=2 \pi f$. Assuming that amplitudes are Gaussian in all three directions, leads to the following equation for the displacement of dark matter, $d$, from its initial static location, $(x, y, z)$, at an instant in time with the center of the photon at the origin:

$$
d(x, y, z)=A_{0} \sin (k x) \mathrm{e}^{\left(-x^{2} / \sigma_{x}^{2} \lambda^{2}\right)} \mathrm{e}^{\left(-y^{2} / \sigma_{y}^{2} \lambda^{2}\right)} \mathrm{e}^{\left(-z^{2} / \sigma_{z}^{2} \lambda^{2}\right)}
$$

where $A_{0}$ is the peak displacement at the center of the photon, $k$ is the wavenumber $(2 \pi / \lambda), \sigma_{i}$ is the standard deviation in the $i$ direction, and $\lambda$ is the wavelength. $\sigma_{i}$ is a unitless number since the size of the photon should logically scale with wavelength. This equation assumes the photon propagation is in the $x$ direction and the $x-y$ plane is the vibration plane, i.e., $d$ is in the $+y$ direction.

The velocity of the dark-matter particle, $v$, is then given by:

$$
v(x, y, z)=A_{0} 2 \pi f \cos (k x) \mathrm{e}^{\left(-x^{2} / \sigma_{x}^{2} \lambda^{2}\right)} \mathrm{e}^{\left(-y^{2} / \sigma_{y}^{2} \lambda^{2}\right)} \mathrm{e}^{\left(-z^{2} / \sigma_{z}^{2} \lambda^{2}\right)} .
$$

The energy in a magnetic field, $E_{B}$, in cgs units [1] is:

$$
E_{B}=\frac{1}{8 \pi} \int \boldsymbol{B} \cdot \boldsymbol{H} \mathrm{d} V
$$


Since the total electromagnetic energy of the photon is twice the magnetic field energy:

$$
E_{B+E}=\frac{1}{4 \pi} \int \boldsymbol{B} \cdot \boldsymbol{H} \mathrm{d} V .
$$

Substituting the equation for velocity, Equation (3), into the equation for the magnetic field, Equation (1), using $B=\mu H$ and solving the integral provides the following relationship for the model parameters:

$$
E_{B+E}=\frac{1}{4} \sqrt{\frac{\pi}{2}} \pi^{2} \mu b^{2} A_{0}^{2} \sigma_{x} \sigma_{y} \sigma_{z} \lambda .
$$

This result indicates that the electromagnetic energy of the photon is proportional to $\lambda$, not $f$ as expected. Thus the model would seem to be in error, however, there is also kinetic energy that is not included in this calculation. In addition, the electromagnetic energy increases as wavelength increases (or frequency decreases), the opposite of the known properties of the photon.

If the electromagnetic energy is small compared to the kinetic energy, then the kinetic energy of the photon is just like the energy of the phonon and explains why both vibrations obey the same equations for energy, momentum, and speed.

Estimates of the model parameters can give some insight into the photon energy as a function of frequency. The magnetic charge, $b$, must be small enough that common motions through dark matter would not be detected as a change in the background field. The fastest velocities where magnetic fields are measured are on some low-Earth-orbit satellites where the detected magnetic field is used to determine attitude. Assuming an orbital velocity of $6.3 \times 10^{5} \mathrm{~cm} \cdot \mathrm{s}^{-1}$ and a maximum magnetic field that would not disrupt the attitude calculation of 0.01 gauss, yields $b<500$ gauss. $A_{0} 2 \pi f$ is the peak velocity of a dark-matter particle at the center of the photon model. If the maximum velocity is assumed to be less than $c / 100$ for the highest energy photon $(100 \mathrm{TeV})$, then $A_{0}<2 \times$ $10^{-21} \mathrm{~cm}$. The size of the photon needs to be large enough to explain diffraction through a dual slit. Dual slits with separation of $1000 \lambda$ and many diffraction peaks have been observed, leading to estimates for the $\sigma$ 's on the order of $10^{3}$ orthogonal to the propagation direction and possibly $10^{2}$ in the propagation direction; leading to $\sigma_{x} \sigma_{y} \sigma_{z}$ being on the order of $10^{8}$. Inserting these estimates and equating the electromagnetic energy to $h f$ yields the point where the electromagnetic energy would equal the traditional value for the photon. The result is $\lambda=8 \times$ $10^{5} \mathrm{~cm}(37 \mathrm{kHz})$; at wavelengths larger than this, the electromagnetic energy dominates the photon energy and at wavelengths less than this, the kinetic energy dominates. The estimates for $b$ and $A_{0}$ are both upper bounds, so it is quite possible that this effect occurs at much larger wavelengths; but these estimates indicate that the effect might be detected in the very low frequency (VLF) band.

The photon is an obvious quantum state. Consider the creation of a photon from the electron transition in an atom. The atom is about $10^{-8} \mathrm{~cm}$ in size, yet creates photons many orders of magnitude larger. If the creation and collapse are mirror images of each other, then the photon starts as a point and ends as a point, yielding the particle-like nature of the photon. Hence, the photon has the properties of a wave until its collapse, when its energy and momentum converge at a point and appear like a particle.

Overall, this analysis indicates that the photon:

- Is considerably larger than the wavelength,

- Is the same as a phonon, but is a vibration in dark matter instead of normal matter,

- Has an electromagnetic energy that is negligible, except at very long wavelengths, and

- Is a quantum state whose creation and collapse provide the particle-like characteristics, while the steady state provides the wave-like characteristics.

\section{Dark-Matter Properties}

The dark-matter particle density can be estimated from the highest energy gamma rays. At the highest possible energy, the wavelength would equal twice the spacing between dark-matter particles. To date the largest gamma ray detected is about $100 \mathrm{TeV}$. (There is no reason to believe that this is the maximum but it can be used to calculate an upper bound for the mass of a dark-matter particle.) This implies an upper limit on the spacing between dark-matter particles is about $6.2 \times 10^{-19} \mathrm{~cm}$, which is significantly smaller than a nucleus, and a dark-matter particle density greater than $4.2 \times 10^{54} \mathrm{~cm}^{-3}$. If a dark-matter density of $2.3 \times 10^{-30} \mathrm{~g} \cdot \mathrm{cm}^{-3}$ is assumed, then the upper bound on the mass of a dark-matter particle is about $5.5 \times 10^{-85} \mathrm{~g}$. These are extraordinary numbers! Even the annihilation of a dark-matter particle is too small to be detectable and the creation of a 
dark-matter particle requires so little energy that they could be abundantly created and not be noticed (even in normal chemical processes). These values for dark-matter properties helps to understand why a dark-matter particle may be so impossible to detect individually and why they could be perceived as a field since only their aggregate properties can be detected and measured.

These properties may also indicate why magnetic charge has not been detected on other particles. The darkmatter density is thirty-some orders of magnitude greater than Avogadro's number and no other particles are available in the same quantities as dark matter.

\section{Speed of Light}

The properties of photons can be inferred by analogy to phonons. The speed of a phonon, the speed of sound, is proportional to $T^{1 / 2}$ in an ideal gas. Assuming the universe, and specifically the dark matter, is expanding adiabatically like an ideal gas [2]:

$$
p^{(1-\gamma)} T^{\gamma}=\text { constant }
$$

where $p$ is pressure, $T$ is temperature, and $\gamma=5 / 3$ for a monatomic gas. The Ideal Gas Law, $p V=n R T$, leads to $p$ being proportional to $\rho T$, where $\rho$ is the dark-matter density. Substituting $\rho T$ into the adiabatic equation leads to $\rho^{1 / 3}$ being proportional to $T^{1 / 2}$. Thus, if the dark matter is similar to an ideal gas, the velocity of light is proportional to $\rho^{1 / 3}$. Therefore, as the universe expands and the dark-matter density decreases, the velocity of light should decrease. This would be an explanation for why the fine structure constant may have been smaller in the past as others have suggested.

There is also the possibility that the dark-matter density is not constant throughout the universe since we know that it is affected by gravity. The density of dark matter would logically be higher at the center of the universe and decrease to the edge of the universe. The dark-matter density would also be logically higher near massive objects than further away. In each of these situations a faster speed of light would occur where the dark matter is denser and slower where the dark matter is less dense. (Maybe this density variation is enough to explain the Pioneer anomaly as the speed of light may be slightly higher near the Sun and slightly lower at further distances from the Sun.) This will have a significant effect on the calculations for the size and age of the universe.

From the analysis above, the speed of light can be given by:

$$
c=c_{o} \sqrt[3]{\rho / \rho_{o}} .
$$

Here $c_{o}$ is the speed of light at a reference point, $\rho$ is the dark-matter density, and $\rho_{o}$ is the dark-matter density at the same reference point. For this paper, the reference point is here at Earth.

\section{Special Relativity}

Einstein's Theory of Special Relativity is based on the speed of light being the same for all observers, and that the laws of physics are the same for observers that are traveling at a constant velocity with respect to each other. In contrast, this dark-matter theory basically says that the speed of light is defined by the dark-matter density and is relative to the dark matter, but this theory can be shown to be consistent with the derivation of the Special Theory of Relativity.

Consider a classical derivation where the rest frame is the dark-matter rest frame. Assume an apparatus where a photon is transmitted to a mirror a distance $d$ away and reflected back to a receiver that is coexistent with the transmitter. The time of flight for the photon is thus $2 d / c$ when it is at rest. Now, move the apparatus with a velocity, $v$, in a direction orthogonal to its optical path. If the experiment is repeated while the apparatus is moving, the observer in the dark-matter reference frame sees that path length increased because of the relative motion. Specifically, the dark-matter observer sees a path length of $2\left(d^{2}+v^{2} t^{2}\right)^{1 / 2}$, where $t$ is the time it takes to arrive at the mirror. An observer moving with the apparatus will see a path length of $2 d$. Dividing both path lengths by $c$ yields the time of flight in each frame of reference. Solving for $t$ in the dark-matter frame yields a time of flight of $4 d^{2} / c^{2} /\left(1-v^{2} / c^{2}\right)^{1 / 2}$. Assuming $c$ is the same for both observers, leads to the standard expression for time dilation $t=t^{\prime} /\left(1-v^{2} / c^{2}\right)^{1 / 2}$, where $t^{\prime}$ is the time for the observer in motion. There is nothing new here other than the derivation specifically assumed a dark-matter sea for the observer at rest and the photon travelling 
at $c$ in this frame.

If the speed of light for the moving observer had not been assumed to be the same, then the logical value to assume would have been the speed of light that the observer at rest would have calculated for the moving reference frame, namely $c\left(1-v^{2} / c^{2}\right)^{1 / 2}$, which is the projection of the speed of light normal to the velocity. However, substituting that into various equations for physical phenomena would yield results that are in conflict with themselves. The only value for the speed of light that leads to the laws of physics remaining the same in both reference frames is $c$. Hence, the assumption that the speed of light is the same for all observers is unnecessary. Accordingly, the assumption that the laws of physics are invariant, is sufficient and a consequence is that the observed speed of light is the same for all reference frames.

\section{Dark-Matter Relativity}

Dark matter has a similarity with special relativity in that the speed of light would appear to be different from other reference frames. As the dark-matter density changes, a logical position would be that the laws of physics do not change, just as Einstein postulated for special relativity. After all, as the universe expands, the density would logically decrease, and from an external observer, the velocity of light would also logically decrease. A decrease in the velocity of light has not been noted in our reference frame but that may be due to a density variation that is not detectable. Plus, if mass is assumed constant, then the speed of light would need to remain the same to keep the rest mass energy the same. (If the mass were assumed to change with density, there are inconsistent results for length and time variations when considering momentum and kinetic energy.) Thus, Einstein's original assertion that the speed of light is the same for all observers is valid for variations in dark-matter density. Since the energy and momentum of a photon should be the same as the dark-matter density changes, then the wavelength and frequency are the same.

Consider a box with a dark-matter density of $\rho$ inside the box and $\rho_{o}$ outside. Thus, to an outside observer the speed of light inside the box is $c_{o}\left(\rho / \rho_{o}\right)^{1 / 3}$. Consider a photon of wavelength $\lambda_{o}$, transmitted parallel to the length of the box. As the photon enters the box its leading edge's speed changes while its trailing edge is unchanged until it is also inside the box. The result is that the photon wavelength as seen from outside the box becomes $\lambda_{o}\left(\rho / \rho_{o}\right)^{1 / 3}$. Since the wavelength as seen by an inside observer must be $\lambda_{o}$, the resulting length variation inside, $l$, relative to outside, $l_{o}$, is given by

$$
l=l_{o} \sqrt[3]{\rho / \rho_{o}} .
$$

Thus the length of an object appears to be proportional to the cube root of the dark-matter density.

Similarly, the photon period is proportional to wavelength, thus the period is also proportional to the cube root of the dark-matter density. Hence, the time inside the box, $t$, relative to outside the box, $t_{o}$, is given by

$$
t=t_{o} \sqrt[3]{\rho / \rho_{o}} .
$$

With both length and time proportional to the cube root of the dark-matter density, velocity is the same to observers both inside and outside the box. Thus, a particle entering the box has the same momentum and kinetic energy observed inside the box as observed outside.

The ramifications are that an observer will not detect changes in the dark-matter density in its surroundings. Observers can sense changes in the speed of light in other reference frames but not in their own. In the limit when dark-matter density goes to zero, dimensions go to zero and time stops; dark matter not only defines the speed of light but it also defines space and time. Without dark matter, space and time would not exist as we know them. At lower densities, objects are smaller and time moves slower. At higher densities, objects are larger and time moves quicker. At the moment after the big bang, the speed of light would have been almost singular and time would have passed in an instant — this, of course, being observed from our or a similar reference frame. Many physical phenomena have been theorized to have occurred in ridiculously short periods of time after the big bang and this may explain why they appear that way from our reference frame.

\section{Nuclear Stability}

The assumptions presented here for dark matter have effects beyond astrophysics and the success of any theory 
depends on its validity in all situations. There is one naturally occurring situation where dark matter may be absent, specifically in the nucleus of an atom where the nucleons are tightly compressed together. The coulombic force is transmitted by virtual photons and virtual photons need the same medium as real photons, then if there is no dark matter, there is no coulombic force. If the nucleons are so tightly packed that dark matter is absent in the nucleus, then this theory provides a fundamental explanation for why the protons do not repel each other in the nucleus - in effect, the coulombic force is turned off.

One can also imagine the surface of large nuclei as being rougher than that of smaller nuclei and rough enough that dark matter could exist between protons protruding above the surface. This would enable the coulombic force to be transmitted between these protons to generate a force (an instability) and expel some protons or alpha particles. This might explain the limit in naturally occurring nuclei and why some isotopes are unstable.

The absence of dark matter also stops time. This can explain why neutrons in the nucleus are stable.

\section{Expansion Rate of the Universe}

If the density of dark matter is largest near large masses, then it is logical that the spaces between large masses, i.e., intergalactic space, would have lower dark-matter densities and a lower speed of light. Tangherlini [3] has calculated using several methods that an index of refraction of intergalactic space of 1.46 - 1.53 can explain the apparent accelerating universe. Since a value greater than one for the index of refraction slows the velocity of light, a similar value for the speed of light can be calculated from a lower density of dark matter. Assuming a value of 1.46 for the index of refraction leads to the same velocity of light if the dark-matter density in intergalactic space were $32 \%$ of the dark-matter density at Earth, a very reasonable value. Hence, this theory supports a more logical conclusion that the universe is decelerating in size.

\section{Blackbody Radiation}

Classically, blackbody radiation has been the transformation of a phonon into a photon, i.e., a transformation of vibrational energy into an electromagnetic wave/particle. To date, how a simple vibration (thermal energy) is turned into an electromagnetic wave/particle seemed to be a complicated task to occur naturally at a boundary. However, it is easy to picture a vibration of matter being transferred to a vibration of dark matter; especially since the dark matter fills the spaces between particles of ordinary matter in a solid and both particles/waves have the same energy and frequency, but not the same speed or wavelength. Thus a photon is emitted with the energy and momentum of the phonon, and the electromagnetic properties are a result of the vibration of the dark-matter's magnetic charge.

\section{Superconductivity}

A very interesting property of superconductors is their expulsion of magnetic fields. The theory addressed in this paper would interpret this as the inability to have a dark-matter current in a superconductor. Hence, inside the superconductor, the dark matter is bound in some state that allows virtual photons to flow while preventing dark-matter currents; this possibly reveals some form of a bound state for dark matter.

While this is very interesting theoretically, the most exciting part may be the ability to produce volumes of space with varying dark-matter densities possibly even producing a dark-matter vacuum. Consider a hollow superconducting tube, where currents are induced in each end, such that the magnetic field lines are going out of both ends. This would expel dark matter from inside the tube through each end and reduce the dark-matter density inside the tube. The effect would prevent the flow of light through the tube if all of the dark matter were expelled. (If it is only partially expelled, the speed of light would be reduced.) As discussed under the Nuclear Stability section, a dark-matter vacuum would prohibit the transmission of virtual photons and the coulomb force would be turned off. Thus, atoms in this dark-matter vacuum would become a plasma because the electrons are no longer bound to their nuclei.

\section{Nuclear Fusion}

An even more exciting possibility would happen if this dark-matter vacuum could be generated (or even partially generated). A dark-matter vacuum would create a perfect space for nuclear fusion to occur, since much less energy would be required to overcome the coulomb repulsion between nuclei. It may also help to explain fusion 
in the sun, if the dark-matter density is considerably reduced or absent at the sun's center.

\section{Tachyons}

The combination of relativistic particles and variable dark-matter densities provides a possible opportunity to create a tachyon. Suppose you have a box where the dark-matter density is lower than outside of the box. A relativistic particle can be generated outside of the box with a velocity that is greater than the speed of light inside the box. If the particle is injected into the box, an observer outside the box would think that the particle inside the box is travelling faster than the light. An observer inside the box would see the particle as having the same velocity as an observer outside the box saw before it entered the box. The observer inside the box would see the particle as having the same energy and momentum as the observer outside the box before it entered the box. A paradox occurs in that the observer outside the box would say that the particle would transverse the box faster than a photon, but the observer inside the box would say that the photon transverses the box faster than the particle.

\section{Multiple Universes}

An interesting situation may exist with this theory. If our universe is the only one caused by our big bang and the big bang created the dark matter, other universes may exist beyond where our dark matter stops. If there is no dark matter connecting these universes, then there would be no transmission of photons between them until they expand to the point where their dark-matter seas start to overlap. Any nondark matter that exists between these universes would exist as a cold plasma.

\section{General Thoughts}

Considerable effort went into trying to find a photon model where its total electromagnetic energy was proportional to $f$. For a photon to have an electromagnetic energy proportional to $f$, its magnetic field needs to be proportional to $f^{2}$ (from Equation (5) and the assumption that a characteristic volume is proportional to $\lambda^{3}$ ). If $\boldsymbol{B}$ were assumed to be proportional to $v^{2}$, then the energy would be proportional to $f$, however, the model parameters are not reasonable. Assuming higher powers of $\boldsymbol{v}$ and an amplitude that is a function of $f$, can provide energies proportional to $f$, but again the model parameters are not reasonable. In addition, in each of these higher power cases, the magnetic field is not a pure sinusoid. In each of these cases, a sinusoidal vibration was assumed. Other vibration functions can be assumed to achieve a sinusoidal field but these functions would be illogical. As a result, the model presented in the Photon section was the simplest with a sinusoidal vibration, leading to a sinusoidal magnetic field but an energy proportional to $\lambda$. The model choices to select the best were between: a nonsinusoidal magnetic field, a nonsinusoidal vibration, or an electromagnetic energy that was insignificant with respect to the kinetic energy. The ability to show that the electromagnetic energy was insignificant to the vibrational energy made the selected choice the most logical.

The magnetic field was assumed to be proportional to the velocity of a dark-matter particle. In reality, the field is probably at a peak at the dark-matter particle and reduced at farther distances. However, the extraordinary density of particles and an effective short range would indicate that the assumption used in the model is equivalent to the sum of all fields from the nearby dark-matter particles and provides the same result without knowing the exact function for the field from a single particle.

A simple experiment can determine if this theory, or a more complicated one, is correct. Consider a coherent laser signal split between two fibers and a solenoid. String one of the fibers multiple times through the solenoid, such that the light travels parallel to the solenoid's magnetic field. String the other fiber multiple times through the solenoid such that the light travels against the solenoid's magnetic field. As the solenoid is energized, the speed of light in one fiber is slowed while the speed of light in the other is sped up due to the dark-matter currents produced by the solenoid (assuming these fields are present inside the fibers). The result should be a phase difference between the two fibers as voltage is applied to the solenoid.

The photon model discussed here is based on a transverse wave in dark matter. Longitudinal waves should also be possible that could communicate another force. Such a wave would have an oscillating magnetic field in the propagation direction and, from Faraday's Law, an electric field that rotates around the propagation direction. Such a wave would resemble a circularly polarized photon. 
The Earth's magnetic field looks much like a magnetic dipole field, except for the distortion due to solar wind. If the magnetic field is a current of dark matter, the physical interaction with the current of particles from the Sun could create the pattern observed, especially since the distortion occurs with the weakest dark-matter currents.

Sun spots may be another opportunity to observe effects of dark matter. Could the dark spots be a result of the annihilation of dark matter or the dark matter being blown away, in either case leaving a dark-matter vacuum preventing the transmission of photons?

Suppose we have a box where we can control the dark-matter density. If we reduce the density, anything inside the box would age more slowly; basically the box would be a stasis chamber. If the density were increased, then anything in the box would age quicker, making it a very useful tool in growing replacement parts from genetic material.

\section{Conclusions}

A theory has been presented for dark matter that leads to dark matter appearing to be the ether that was abandoned a century ago while providing plausible explanations for some physical phenomenon. The theory:

- Assumes the existence of a magnetic charge on dark matter that adds symmetry to Maxwell's equations with the addition of magnetic-monopole-like terms,

- Leads to a model for the photon that implies its energy is largely kinetic, except at very low energies, and that exhibits particle-wave duality,

- Explains the apparent acceleration of the universe when it is actually decelerating,

- Provides an explanation for nuclear stability, and

- Leads to dark matter being necessary for space and time to exist.

The theory also provides insights into other phenomenon and several opportunities for major breakthroughs in other scientific areas if the dark-matter density can be manipulated.

The debate on the existence of an ether should be reopened with the additional knowledge available today.

\section{References}

[1] Jackson, J.D. (1962) Classical Electrodynamics. John Wiley \& Sons, Inc., New York, 162.

[2] Sears, F.W. (1964) An Introduction to Thermodynamics, the Kinetic Theory of Gases, and Statistical Mechanics. 2nd Edition, Addison-Wesley Publishing Company, Inc., Reading, Massachusetts, 69.

[3] Tangherlini, F.R. (2015) Journal of Modern Physics, 6, 1360-1370. http://dx.doi.org/10.4236/jmp.2015.69141 